\title{
Right- and left-sided colorectal cancers respond differently to cetuximab
}

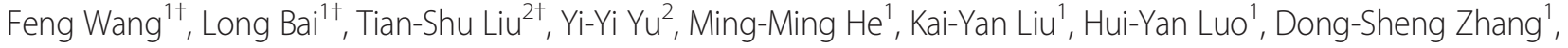 \\ Yin Jin ${ }^{1}$, Feng-Hua Wang ${ }^{1}$, Zhi-Qiang Wang ${ }^{1}$, De-Shen Wang ${ }^{1}$, Miao-Zhen Qiu', Chao Ren ${ }^{1}$, Yu-Hong Li ${ }^{\text {* }}$ \\ and Rui-Hua Xu* ${ }^{*}$
}

\begin{abstract}
Introduction: Right-sided colon cancer (RSCC) and left-sided colorectal cancer (LSCRC) differ with respect to their biology and genomic patterns. This study aimed to examine whether the primary tumor location is associated with the response to cetuximab in patients with metastatic colorectal cancer (mCRC).

Methods: Patients with $\mathrm{mCRC}$ treated with cetuximab and standard chemotherapy as first- or second-line treatments were compared with randomly chosen patients who were treated with chemotherapy alone between 2005 and 2013. The main outcome measures were the overall response rate (ORR), progression-free survival (PFS), and overall survival (OS). The differences in the outcome were analyzed by using the chi-squared test, Student's $t$ test, and Kaplan-Meier method.

Results: The treatment results of 206 patients with mCRC treated with cetuximab and standard chemotherapy as first- or second-line treatments were compared with those of 210 patients who were treated with chemotherapy alone. As a first-line treatment, cetuximab with chemotherapy was associated with a significantly higher ORR ( $49.4 \%$ vs. $28.6 \%, P=0.005$ ) as well as longer PFS (9.1 vs. 6.2 months, $P=0.002$ ) and OS (28.9 vs. 20.1 months, $P=0.036)$ than chemotherapy alone in patients with LSCRC. However, cetuximab neither improved the ORR ( $36.4 \%$ vs. $26.2 \%, P=0.349$ ) nor prolonged PFS (5.6 vs. 5.7 months, $P=0.904)$ or OS ( 25.1 vs. 19.8 months, $P=0.553$ ) in patients with RSCC. As a second-line treatment, cetuximab exhibited a tendency to improve the ORR ( $23.5 \%$ vs. $10.2 \%$, $P=0.087$ ) and prolong PFS (4.9 vs. 3.5 months, $P=0.064)$, and it significantly prolonged OS (17.1 vs. 12.4 months, $P=0.047)$ compared with chemotherapy alone in the patients with LSCRC. In contrast, as a second-line treatment, cetuximab neither improved the ORR (7.1\% vs. $11.4 \%, P=0.698)$ nor prolonged PFS (3.3 vs. 4.2 months, $P=0.761)$ or OS (13.4 vs. 13.0 months, $P=0.652$ ) in patients with RSCC.
\end{abstract}

Conclusions: The addition of cetuximab to chemotherapy in both first- and second-line treatments of mCRC may only benefit patients with primary LSCRC.

Keywords: Colorectal neoplasms, Cetuximab, Left-sided, Right-sided, Chemotherapy

\section{Background}

Colorectal carcinomas (CRCs) that occur proximal (right) or distal (left) to the splenic flexure exhibit differences in their embryologic development, blood supply, macroscopic pathology, and clinicopathologic parameters $[1,2]$. The right colon arises from the embryonic midgut

\footnotetext{
* Correspondence: liyh@sysucc.org.cn; xurh@sysucc.org.cn

${ }^{\dagger}$ Equal contributors

'Department of Medical Oncology, Sun Yat-sen University Cancer Center: State Key Laboratory of Oncology in South China; Collaborative Innovation Center for Cancer Medicine, 651 Dongfeng Road East, Guangzhou, Guangdong 510060, P. R. China

Full list of author information is available at the end of the article
}

and is perfused by the superior mesenteric artery, whereas the left colon originates from the hindgut and is served by the inferior mesenteric artery [2]. Right-sided colon cancers (RSCCs) are typically bulky, exophytic, polypoid lesions that project into the lumen and cause significant anemia, whereas left-sided colorectal cancers (LSCRCs) are typically infiltrating, constricting lesions that encircle the lumen, often leading to obstruction [1]. Poorly differentiated adenocarcinoma or signet-ring cell carcinoma and mucinous adenocarcinoma are more frequently seen in the right colon than in the left colon [3]. RSCCs typically present at a more advanced stage, and the patients 
with RSCCs have a significantly worse survival than patients with LSCRCs [4-6].

Recent studies have revealed distinguishable genomic patterns between LSCRC and RSCC [7, 8]. LSCRCs exhibit higher p53 gene mutation and cyclooxygenase-2 expression rates with more common chromosomal instability [9-12]. RSCCs are generally diploid, and they exhibit higher rates of microsatellite instability (MSI) and higher expression of cytoplasmic c-erbB2 and epidermal growth factor receptors (EGFRs) [13]. Recent whole genome analysis has shown that RSCCs are more likely to be hypermethylated as well as to have elevated mutation rates compared with LSCRCs [7]. Some studies have reported significantly more activating mutations in codon $12 / 13$ of Kirsten rat sarcoma viral oncogene homolog (KRAS) in RSCCs than in LSCRCs [14, 15]. However, other studies have reported that there is no substantial difference in the KRAS mutations between RSCCs and LSCRCs $[8,16]$. LSCRCs exhibit a significant association between KRAS activation and distant organ metastasis, whereas RSCCs do not. Mutation of KRAS was found to be associated with a significantly poorer prognosis in patients with LSCRCs, but not in those with RSCCs [17].

Cetuximab is a chimeric IgG1 monoclonal antibody that binds to the extracellular domain of EGFR; also, it blocks ligand-induced receptor signaling and induces immune-mediated antitumor mechanisms, such as antibody-dependent cell-mediated cytotoxicity [18, 19]. Cetuximab is currently approved for the treatment of

Table 1 Baseline clinicopathologic characteristics of patients with first-line treatment

\begin{tabular}{|c|c|c|c|c|c|c|c|c|c|}
\hline \multirow[t]{2}{*}{ Clinicopathologic characteristic } & \multicolumn{4}{|c|}{ Cetuximab group } & \multicolumn{4}{|c|}{ Chemotherapy alone group } & \multirow[t]{2}{*}{$P$ value ${ }^{d}$} \\
\hline & All patients & $\mathrm{RSCC}$ & LSCRC & $P$ value & All patients & $\mathrm{RSCC}$ & LSCRC & $P$ value & \\
\hline No. of patients & 110 & 33 & 77 & - & 117 & 61 & 56 & - & $0.001^{*}$ \\
\hline Median age at diagnosis & & & & 0.420 & & & & 0.502 & 0.613 \\
\hline$\leq 65$ years & $90(81.8)$ & $29(87.9)$ & $62(80.5)$ & & $93(79.5)$ & $50(82.0)$ & $43(76.8)$ & & \\
\hline$>65$ years & $20(18.2)$ & $4(12.1)$ & $15(19.5)$ & & $24(20.5)$ & $11(18.0)$ & $13(23.2)$ & & \\
\hline Sex & & & & 0.274 & & & & 0.085 & 0.404 \\
\hline Male & $75(68.2)$ & $20(60.6)$ & $55(71.4)$ & & $73(62.4)$ & $43(70.5)$ & $30(53.6)$ & & \\
\hline Female & $35(31.8)$ & $13(39.4)$ & $22(28.6)$ & & $44(37.6)$ & $18(29.5)$ & $26(46.4)$ & & \\
\hline ECOG performance status & & & & 0.300 & & & & 0.367 & 0.113 \\
\hline 0 & $49(44.5)$ & $11(33.3)$ & $38(49.4)$ & & $49(41.9)$ & $29(47.5)$ & $20(35.7)$ & & \\
\hline 1 & $58(52.7)$ & $21(63.6)$ & $37(48.1)$ & & $57(48.7)$ & $26(42.6)$ & $31(55.4)$ & & \\
\hline$\geq 2$ & $3(2.7)$ & $1(3.0)$ & $2(2.6)$ & & $11(9.4)$ & $6(9.8)$ & $5(8.9)$ & & \\
\hline Pathology & & & & $0.003^{*}$ & & & & 0.116 & 0.608 \\
\hline Adenocarcinoma & $101(91.8)$ & $26(78.8)$ & $75(97.4)$ & & $110(94.0)$ & $55(90.2)$ & $55(98.2)$ & & \\
\hline Mucinous \& signet-ring cell & $9(8.2)$ & $7(21.2)$ & $2(2.6)$ & & $7(6.0)$ & $6(9.8)$ & $1(1.8)$ & & \\
\hline Metastasis & & & & 0.674 & & & & $0.015^{*}$ & 0.893 \\
\hline Single & $67(60.9)$ & $19(57.6)$ & $48(62.3)$ & & $70(59.8)$ & $43(70.5)$ & $27(48.2)$ & & \\
\hline Multiple & $43(39.1)$ & $14(42.4)$ & $29(37.7)$ & & $47(40.2)$ & $18(29.5)$ & $29(51.8)$ & & \\
\hline Metastasis resection & $29(26.4)$ & $6(18.2)$ & $23(29.9)$ & 0.244 & $28(23.9)$ & $15(24.6)$ & $13(23.2)$ & 1.000 & 0.760 \\
\hline Total cycles of cetuximab ${ }^{a}$ & $8(2-29)$ & $9(2-29)$ & $8(2-29)$ & 0.537 & - & - & - & - & - \\
\hline Total cycles of chemotherapy ${ }^{a}$ & $13(2-43)$ & $13(3-38)$ & $13(2-43)$ & 0.928 & $13(2-38)$ & $14(2-38)$ & $13(4-38)$ & 0.546 & 0.344 \\
\hline All three active drugs ${ }^{b}$ & $73(66.4)$ & $24(72.7)$ & $49(63.6)$ & 0.388 & $89(76.1)$ & $48(78.7)$ & $41(73.2)$ & 0.522 & 0.109 \\
\hline Backbone chemotherapy & & & & $0.029^{*}$ & & & & $0.041^{*}$ & $0.003^{*}$ \\
\hline Oxaliplatin-based & $37(33.6)$ & $6(18.2)$ & $31(40.3)$ & & $63(53.8)$ & $27(44.3)$ & $36(64.3)$ & & \\
\hline Irinotecan-based & $73(66.4)$ & $27(81.8)$ & $46(59.7)$ & & $54(46.2)$ & $34(55.7)$ & $20(35.7)$ & & \\
\hline Bevacizumab during the disease & $44(40.0)$ & $15(45.5)$ & $29(37.7)$ & 0.525 & $38(32.5)$ & $24(39.3)$ & $14(25.0)$ & 0.116 & 0.270 \\
\hline Recurrent disease ${ }^{c}$ & $31(28.2)$ & $6(18.2)$ & $25(32.5)$ & 0.230 & $32(27.4)$ & $18(29.5)$ & $14(25.0)$ & 0.679 & 1.000 \\
\hline
\end{tabular}

${ }^{a}$ The values are presented as median followed by range in parentheses; other values are presented as the number of patients followed by percentages in parentheses. ${ }^{b}$ Patients who received 5 -fluorouracil, oxaliplatin, and irinotecan during the course of their disease. ${ }^{\mathrm{C}}$ Patients who had metastatic disease after curative-intent primary tumor resection. ${ }^{d}$ Patient characteristics were compared between the cetuximab and chemotherapy groups. ECOG, Eastern Cooperative Oncology Group; RSCC, right-sided colon cancer; LSCRC, left-sided colorectal cancer 
metastatic CRC with wild-type KRAS as a monotherapy or in combination with chemotherapy in first-, second-, or third-line settings [20]. Only patients with metastatic colorectal cancer (mCRC) expressing wild-type KRAS respond to cetuximab and show improvements in progression-free survival (PFS) and overall survival (OS) [21]. KRAS mutations and the B-Raf proto-oncogene, serine/threonine kinase (BRAF) V600E mutation, the key molecules of the epidermal growth factor (EGF) pathway, are predictors of resistance to cetuximab therapy [21-23].

It remains unknown whether the primary tumor location affects the response of $\mathrm{mCRC}$ patients to first- and second-line cetuximab treatments. This retrospective multicenter study aimed to investigate the association of the tumor location with tumor response to cetuximab and survival in mCRC patients.

\section{Patients and methods}

\section{Patients and treatment}

In this retrospective study, we assessed mCRC patients treated with cetuximab plus chemotherapy as a first- or second-line therapy at two national cancer centers (Sun Yat-sen University Cancer Center and Fudan University Cancer Center) between January 2006 and December 2013 (the cetuximab group). Similar numbers of patients with LSCRC and RSCC who received first- or secondline chemotherapy without cetuximab during the same period were included as the chemotherapy alone group by means of isometric mechanical random sampling as control groups. Enrolled patients have received mFOLFOX-6 (oxaliplatin $85 \mathrm{mg} / \mathrm{m}^{2}$ d1, 5-fluorouracil [5FU] bolus $400 \mathrm{mg} / \mathrm{m}^{2} \mathrm{~d} 1,5-\mathrm{FU} 2,400 \mathrm{mg} / \mathrm{m}^{2}$ continuous infusion for 46 hours every 2 weeks), XELOX (oxaliplatin

Table 2 Baseline clinicopathologic characteristics of patients with second-line treatment

\begin{tabular}{|c|c|c|c|c|c|c|c|c|c|}
\hline \multirow[t]{2}{*}{ Clinicopathologic characteristic } & \multicolumn{4}{|c|}{ Cetuximab group } & \multicolumn{4}{|c|}{ Chemotherapy alone group } & \multirow[t]{2}{*}{$P$ value } \\
\hline & All patients & RSCC & LSCRC & $P$ value & All patients & $\mathrm{RSCC}$ & LSCRC & $P$ value & \\
\hline No. of patients & 96 & 28 & 68 & - & 93 & 44 & 49 & - & $0.011^{*}$ \\
\hline \multicolumn{10}{|l|}{ Median age at diagnosis } \\
\hline$\leq 65$ years & $82(85.4)$ & $21(75.0)$ & $61(89.7)$ & 0.108 & $72(77.4)$ & $30(68.2)$ & $42(85.7)$ & 0.051 & 0.191 \\
\hline$>65$ years & $14(14.6)$ & $7(25.0)$ & $7(10.3)$ & & $21(22.6)$ & $14(31.8)$ & $7(14.3)$ & & \\
\hline Sex & & & & 0.488 & & & & 1.000 & 0.105 \\
\hline Male & $63(65.6)$ & $20(71.4)$ & $43(63.2)$ & & $50(53.8)$ & $24(54.5)$ & $26(53.1)$ & & \\
\hline Female & $33(34.4)$ & $8(28.6)$ & $25(36.8)$ & & $43(46.2)$ & $20(45.5)$ & $23(46.9)$ & & \\
\hline ECOG performance status & & & & 0.074 & & & & 0.438 & 0.310 \\
\hline 0 & $53(55.2)$ & $13(46.4)$ & $40(58.8)$ & & $41(44.0)$ & $19(43.2)$ & $22(44.9)$ & & \\
\hline 1 & $35(36.5)$ & $11(39.3)$ & $24(35.3)$ & & $42(45.2)$ & $22(50.0)$ & $20(40.8)$ & & \\
\hline$\geq 2$ & $8(8.3)$ & $4(14.3)$ & $4(5.9)$ & & $10(10.8)$ & $3(6.8)$ & $7(14.3)$ & & \\
\hline Pathology & & & & 0.102 & & & & 0.183 & 0.822 \\
\hline Adenocarcinoma & $84(87.5)$ & $22(78.6)$ & $62(91.2)$ & & $83(89.2)$ & $37(84.1)$ & $46(93.9)$ & & \\
\hline Mucinous \& signet-ring cell & $12(12.5)$ & $6(21.4)$ & $6(8.8)$ & & $10(10.8)$ & $7(15.9)$ & $3(6.1)$ & & \\
\hline Metastasis & & & & 0.804 & & & & 1.000 & $0.005^{*}$ \\
\hline Single & $69(71.9)$ & $21(75.0)$ & $48(70.6)$ & & $48(51.6)$ & $23(52.3)$ & $25(51.0)$ & & \\
\hline Multiple & $27(28.1)$ & $7(25.0)$ & $20(29.4)$ & & $45(48.4)$ & $21(47.7)$ & $24(49.0)$ & & \\
\hline Metastasis resection & $24(25.0)$ & $6(21.4)$ & $18(26.5)$ & 0.796 & $16(17.2)$ & $11(25.0)$ & $15(30.6)$ & 0.646 & 0.215 \\
\hline Total cycles of cetuximab (median) & $5(2-20)$ & $4(2-13)$ & $5(2-20)$ & 0.272 & - & - & - & - & - \\
\hline Total cycles of chemotherapy (median) & $8(2-66)$ & $7(4-35)$ & $8(2-66)$ & 0.548 & $6(2-36)$ & $6(2-36)$ & $6(2-27)$ & 1.000 & $0.031^{*}$ \\
\hline All three active drugs ${ }^{a}$ & $87(90.6)$ & $24(85.7)$ & $63(92.6)$ & 0.441 & $84(90.3)$ & 39 (88.6) & $45(91.8)$ & 0.731 & 1.000 \\
\hline Backbone chemotherapy & & & & 0.139 & & & & 1.000 & 0.745 \\
\hline Oxaliplatin-based & $27(28.1)$ & $11(39.3)$ & $16(23.5)$ & & $24(25.8)$ & $11(25.0)$ & $13(26.5)$ & & \\
\hline Irinotecan-based & $69(71.9)$ & $17(60.7)$ & $52(76.5)$ & & $69(74.2)$ & $33(75.0)$ & $36(73.5)$ & & \\
\hline Bevacizumab during the disease & $27(28.1)$ & $11(39.3)$ & $16(23.5)$ & 0.637 & $22(23.7)$ & $8(18.2)$ & $14(28.6)$ & 0.329 & 0.510 \\
\hline Recurrent disease ${ }^{b}$ & $42(43.8)$ & $6(21.4)$ & $36(52.9)$ & $0.006^{*}$ & $33(35.5)$ & 19 (43.2) & $14(28.6)$ & 0.199 & 0.298 \\
\hline
\end{tabular}

All values are presented as the number of patients followed by percentages in parentheses. ${ }^{\text {a }}$ Patients who received 5 -fluorouracil, oxaliplatin, and irinotecan during the course of their disease. ${ }^{b}$ Patients who had metastatic disease after curative-intent primary tumor resection. ${ }^{c}$ Patient characteristics were compared between the cetuximab and chemotherapy groups. Other footnotes as in Table 1 
$130 \mathrm{mg} / \mathrm{m}^{2} \mathrm{~d} 1$, capecitabine $2,000 \mathrm{mg} / \mathrm{m}^{2} \mathrm{~d} 1-14$ every 3 weeks), or modified FOLFIRI (irinotecan $180 \mathrm{mg} / \mathrm{m}^{2} \mathrm{~d} 1$, 5 -FU bolus $400 \mathrm{mg} / \mathrm{m}^{2} \mathrm{~d} 1,5-\mathrm{FU} 2,400 \mathrm{mg} / \mathrm{m}^{2}$ continuous infusion for 46 hours every 2 weeks) and in combination with 5 -FU-based regimens (cetuximab $400 \mathrm{mg} / \mathrm{m}^{2}$ taken at the first dose and followed by $500 \mathrm{mg} / \mathrm{m}^{2}$ every 2 weeks) or capecitabine-based regimens (capecitabine $750 \mathrm{mg} / \mathrm{m}^{2}$ every 3 weeks) in the cetuximab group. All patients had histologically proven mCRC and offered written informed consent for possible future data analysis before treatment. Colon cancers arising in or proximal to the splenic flexure were defined as RSCCs, and those arising distal to the splenic flexure were defined as LSCRCs. Clinicpathologic characteristics (patient age, sex, performance status, and pathologic subtype), curative-intent metastasis resection, treatment duration of chemotherapy, and cetuximab, backbone chemotherapy regimen, exposure to three active chemotherapy agents, exposure to bevacizumab therapy, disease-free survival after curative-intent primary tumor resection, and follow-up data were compared between patients with LSCRCs and those with RSCCs. The tumor response was evaluated by computerized tomodensitometry according to the Response Evaluation Criteria in Solid Tumors (RECIST) version 1.1. Objective response rate (ORR) was defined as percentage of patients showing complete response $(\mathrm{CR})$ or partial response $(\mathrm{PR})$ as best response according to RECIST.

\section{Statistical analysis}

The patient characteristics and response rates were compared by using Student's $t$ test or the chi-square test. The PFS was calculated from the initiation of a first- or second-line treatment to the date of tumor progression,

Table 3 Objective response rate to first-line treatment in patients with metastatic colorectal cancer

\begin{tabular}{lccccc}
\hline Response & RSCC & & & LSCRC \\
\cline { 2 - 3 } \cline { 5 - 6 } & $\begin{array}{l}\text { Chemotherapy } \\
\text { alone group }\end{array}$ & $\begin{array}{l}\text { Cetuximab } \\
\text { group }\end{array}$ & $\begin{array}{l}\text { Chemotherapy } \\
\text { alone group }\end{array}$ & $\begin{array}{l}\text { Cetuximab } \\
\text { group }\end{array}$ \\
\hline Total & 61 & 33 & 56 & 77 \\
Best ORR & $16(26.2)$ & $12(36.4)$ & $16(28.6)$ & $38(49.4)$ \\
CR & $3(4.9)$ & $0(0)$ & $0(0)$ & $1(1.3)$ \\
PR & $13(21.3)$ & $12(36.4)$ & $16(28.6)$ & $37(48.1)$ \\
SD & $28(45.9)$ & $11(33.3)$ & $28(50.0)$ & $33(42.8)$ \\
PD & $14(23.0)$ & $10(30.3)$ & $12(21.4)$ & $4(5.2)$ \\
NA & $3(4.9)$ & $0(0)$ & $0(0)$ & $2(2.6)$ \\
P value ${ }^{\text {a }}$ & 0.349 & & & 0.005 &
\end{tabular}

All values are presented as the number of patients followed by the percentages in parentheses

${ }^{a}$ The ORR to the first-line treatment was compared between the chemotherapy and cetuximab plus chemotherapy groups in RSCC and LSCRC patients, respectively

$\mathrm{ORR}$, objective response rate; $\mathrm{CR}$, complete response; $\mathrm{PR}$, partial response; $\mathrm{SD}$, stable disease; PD, progressive disease; NA, not available. Other footnotes as in Table 1
Table 4 Objective response rates to first- and second-line treatments in patients who received chemotherapy alone

\begin{tabular}{|c|c|c|c|c|}
\hline \multirow[t]{2}{*}{ Response } & \multicolumn{2}{|c|}{$\underline{\text { Fist-line treatment }}$} & \multicolumn{2}{|c|}{ Second-line treatment } \\
\hline & RSCC & LSCRC & RSCC & LSCRC \\
\hline Total & 61 & 56 & 44 & 49 \\
\hline Best ORR & $16(26.2)$ & $16(28.6)$ & $5(11.4)$ & $5(10.2)$ \\
\hline$C R$ & $3(4.9)$ & $0(0)$ & $0(0)$ & $0(0)$ \\
\hline$P R$ & $13(21.3)$ & $16(28.6)$ & $5(11.4)$ & $5(10.2)$ \\
\hline SD & $28(45.9)$ & $28(50.0)$ & $21(47.7)$ & $23(46.9)$ \\
\hline PD & $14(23.0)$ & $12(21.4)$ & $16(36.4)$ & $19(38.8)$ \\
\hline NA & $3(4.9)$ & $0(0)$ & $2(4.5)$ & $2(4.1)$ \\
\hline$P$ value ${ }^{a}$ & \multicolumn{2}{|c|}{0.837} & \multicolumn{2}{|c|}{1.000} \\
\hline
\end{tabular}

a The ORR was compared between the RSCC and LSCRC patients who received chemotherapy alone. Footnotes as in Tables 1 and 3

death from any cause, or the last follow-up. The OS was defined as the duration from the start of a first- or second-line therapy to death of any cause or the date of the last follow-up. All point data were censored. Both the PFS and OS were estimated by using the KaplanMeier method and compared by using the log-rank test. Statistical analysis was performed by using SPSS software 19.0 (SPSS Inc., Chicago, IL, USA). The level of significance was set at $P=0.05$.

\section{Results}

\section{Patient characteristics}

A total of $206 \mathrm{mCRC}$ patients who received combination treatment with cetuximab and chemotherapy as a firstline (110 patients) or second-line (96 patients) therapy were included in this study. In addition, $210 \mathrm{mCRC}$ patients were chosen as controls from those who received first-line (117 patients) or second-line chemotherapy (93 patients) without receiving cetuximab during the same period. The clinicopathologic characteristics of these patients are shown in Tables 1 and 2. In patients

Table 5 Objective response rates of first- and second-line treatments in patients who received cetuximab plus chemotherapy

\begin{tabular}{|c|c|c|c|c|}
\hline \multirow[t]{2}{*}{ Response } & \multicolumn{2}{|c|}{ Fist-line treatment } & \multicolumn{2}{|c|}{ Second-line treatment } \\
\hline & RSCC & LSCRC & RSCC & LSCRC \\
\hline Total & 33 & 77 & 28 & 68 \\
\hline Best ORR & $12(36.4)$ & $38(49.4)$ & $2(7.1)$ & $16(23.5)$ \\
\hline$C R$ & $0(0)$ & $1(1.3)$ & $0(0)$ & $1(1.5)$ \\
\hline PR & $12(36.4)$ & $37(48.1)$ & $2(7.1)$ & $15(22.0)$ \\
\hline SD & $11(33.3)$ & $33(42.8)$ & $16(57.2)$ & $34(50.0)$ \\
\hline PD & $10(30.3)$ & $4(5.2)$ & $10(35.7)$ & $17(25.0)$ \\
\hline NA & $0(0)$ & $2(2.6)$ & $0(0)$ & $1(1.5)$ \\
\hline$P$ value $^{a}$ & \multicolumn{2}{|c|}{0.296} & \multicolumn{2}{|c|}{0.085} \\
\hline
\end{tabular}

${ }^{a}$ The ORR was compared between RSCC and LSCRC patients who received chemotherapy alone. Other footnotes as in Tables 1 and 3 
Table 6 Objective response rates to second-line treatment in patients with metastatic colorectal cancer

\begin{tabular}{|c|c|c|c|c|}
\hline \multirow[t]{2}{*}{ Response } & \multicolumn{2}{|l|}{ RSCC } & \multicolumn{2}{|l|}{ LSCRC } \\
\hline & $\begin{array}{l}\text { Chemotherapy } \\
\text { alone group }\end{array}$ & $\begin{array}{l}\text { Cetuximab } \\
\text { group }\end{array}$ & $\begin{array}{l}\text { Chemotherapy } \\
\text { alone group }\end{array}$ & $\begin{array}{l}\text { Cetuximab } \\
\text { group }\end{array}$ \\
\hline Total & 44 & 28 & 49 & 68 \\
\hline Best ORR & $5(11.4)$ & $2(7.1)$ & $5(10.2)$ & $16(23.5)$ \\
\hline$C R$ & $0(0)$ & $0(0)$ & $0(0)$ & $1(1.4)$ \\
\hline PR & $5(11.4)$ & $2(7.1)$ & $5(10.2)$ & $15(22.1)$ \\
\hline SD & $21(47.7)$ & $16(57.2)$ & $23(46.9)$ & $34(50.0)$ \\
\hline PD & $16(36.4)$ & $10(35.7)$ & 19 (38.8) & $17(25.0)$ \\
\hline NA & $2(4.5)$ & $0(0)$ & $2(4.1)$ & $1(1.5)$ \\
\hline$P$ value $^{a}$ & \multicolumn{2}{|c|}{0.698} & \multicolumn{2}{|c|}{0.087} \\
\hline
\end{tabular}

a The ORR to the second-line treatment was compared between the chemotherapy and cetuximab plus chemotherapy groups of RSCC and LSCRC patients, respectively.

Other footnotes as in Tables 1 and 3 who received first-line therapy, all characteristics except the backbone chemotherapy were well-balanced between the cetuximab group and the chemotherapy alone group (Table 1).

For the first-line therapy, more patients in the chemotherapy alone group received oxaliplatin-based chemotherapy than those in the cetuximab group $(53.8 \%$ vs. $33.6 \%, P=0.003)$. By contrast, in those who received second-line therapy, more patients in the cetuximab group had single organ metastasis $(71.9 \%$ vs. $51.6 \%$, $P=0.005)$ than those in the chemotherapy alone group. In addition, patients in the cetuximab group had a longer duration of chemotherapy administration as secondline therapy ( 8 vs. 6 cycles, $P=0.031$ ) than those in the chemotherapy alone group.

Patients were separated into two subgroups (RSCC and LSCRC) according to the primary tumor location. In the first-line therapy subgroup (Table 1), age, sex, Eastern Cooperative Oncology Group (ECOG) performance status, tumor grade, location of metastasis, resection of the
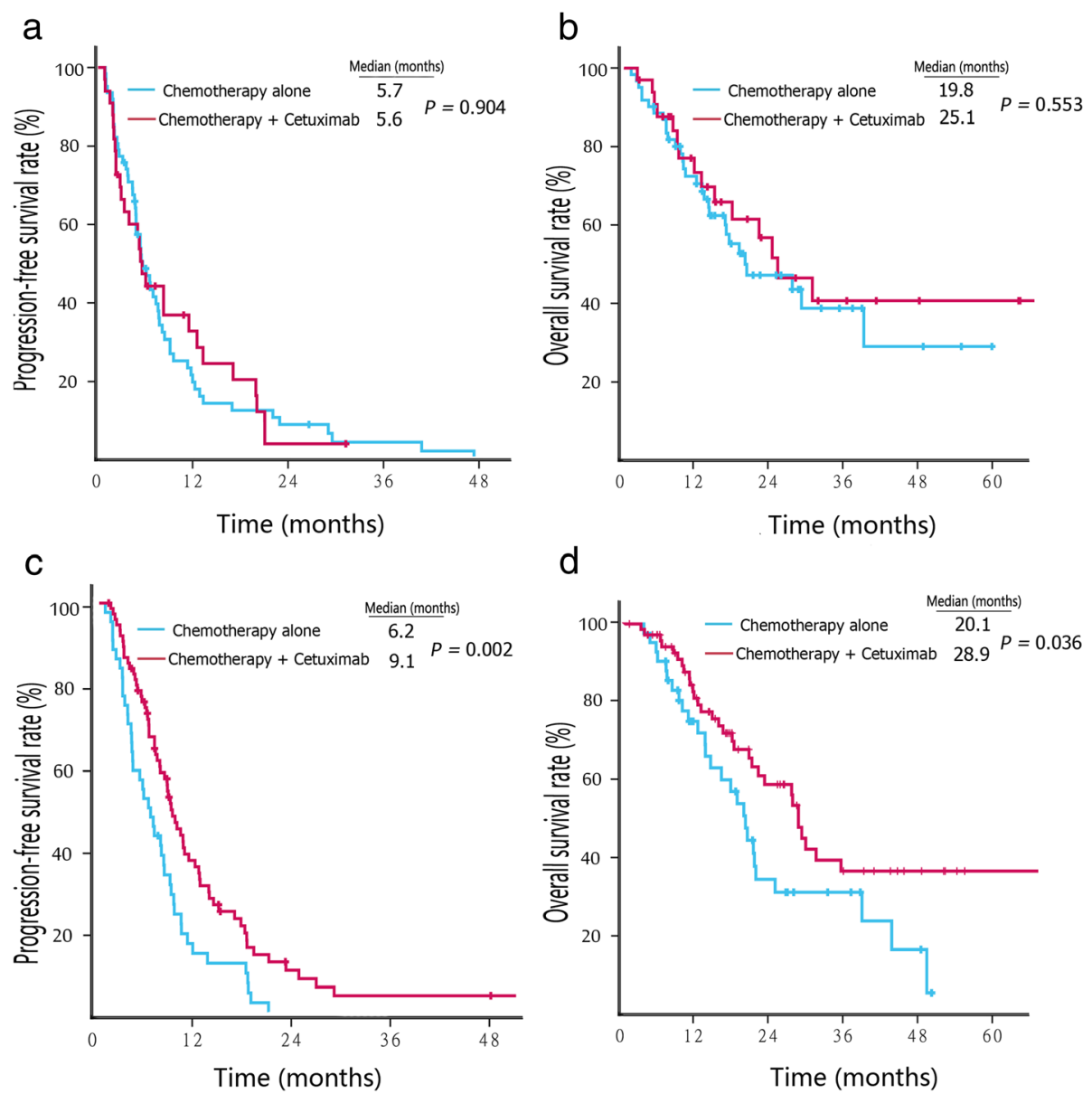

Fig. 1 Kaplan-Meier survival estimates of metastatic colorectal cancer patients with first-line therapy. a, progression-free survival (PFS) curves of right-sided colon cancer (RSCC) patients. b, overall survival (OS) curves of RSCC patients. c, PFS curves of left-sided colorectal cancer (LSCRC) patients. $\mathbf{d}$, OS curves of LSCRC patients 
primary or metastatic site, total cycles of cetuximab or chemotherapy, percentage of three active chemotherapy drugs (5-fluorouracil [FU], oxaliplatin, and irinotecan), treatment with bevacizumab, and recurrent disease did not differ between patients with RSCC and those with LSCRC in both the cetuximab group and the chemotherapy alone group. The proportion of mucinous or signetring cell carcinomas was significantly higher in patients with RSCC than in patients with LSCRC in the cetuximab group ( $21.2 \%$ vs. $2.6 \%, P=0.003)$. The proportion of patients with multiple metastases was significantly higher in patients with LSCRC than in those with RSCC in the chemotherapy alone group ( $51.8 \%$ vs. $29.5 \%, P=0.015)$. More patients with RSCC received irinotecan-based firstline chemotherapy than patients with LSCRC in either the cetuximab group $(81.8 \%$ vs. $59.7 \%, P=0.029)$ or in the chemotherapy alone group ( $55.7 \%$ vs. $35.7 \%, P=0.041)$

\section{ORR}

As a first-line treatment, the addition of cetuximab to chemotherapy significantly improved the ORR compared with chemotherapy alone in patients with LSCRC (49.4 \% vs. $28.6 \%, P=0.005$ ) (Table 3 ). However, the ORR was comparable between the two groups in patients with RSCC ( $36.4 \%$ vs. $26.2 \%, P=0.349)$. There was no significant difference in the response rate between patients with LSCRC and those with RSCC in both the chemotherapy alone group $(28.6 \%$ vs. $26.2 \%$, $P=0.837)$ and the cetuximab group $(49.4 \%$ vs. $36.4 \%$, $P=0.296)$ (Tables 4 and 5 , respectively).

As a second-line treatment, cetuximab with chemotherapy had a tendency to improve the ORR compared with chemotherapy alone in patients with LSCRC (23.5 \% vs. $7.1 \%, P=0.085)$, but not in those with RSCC (7.1 \% vs. $11.4 \%, P=0.698)$ (Table 6). Although there was no difference in response rate between patients with LSCRC and RSCC treated with chemotherapy alone $(10.2 \%$ vs. $11.4 \%, P=1.000)$, cetuximab with chemotherapy exhibited a trend of improving response rate in patients with LSCRC compared with those with RSCC (23.5\% vs. $7.1 \%, P=0.085)$ (Tables 4 and 5 , respectively).
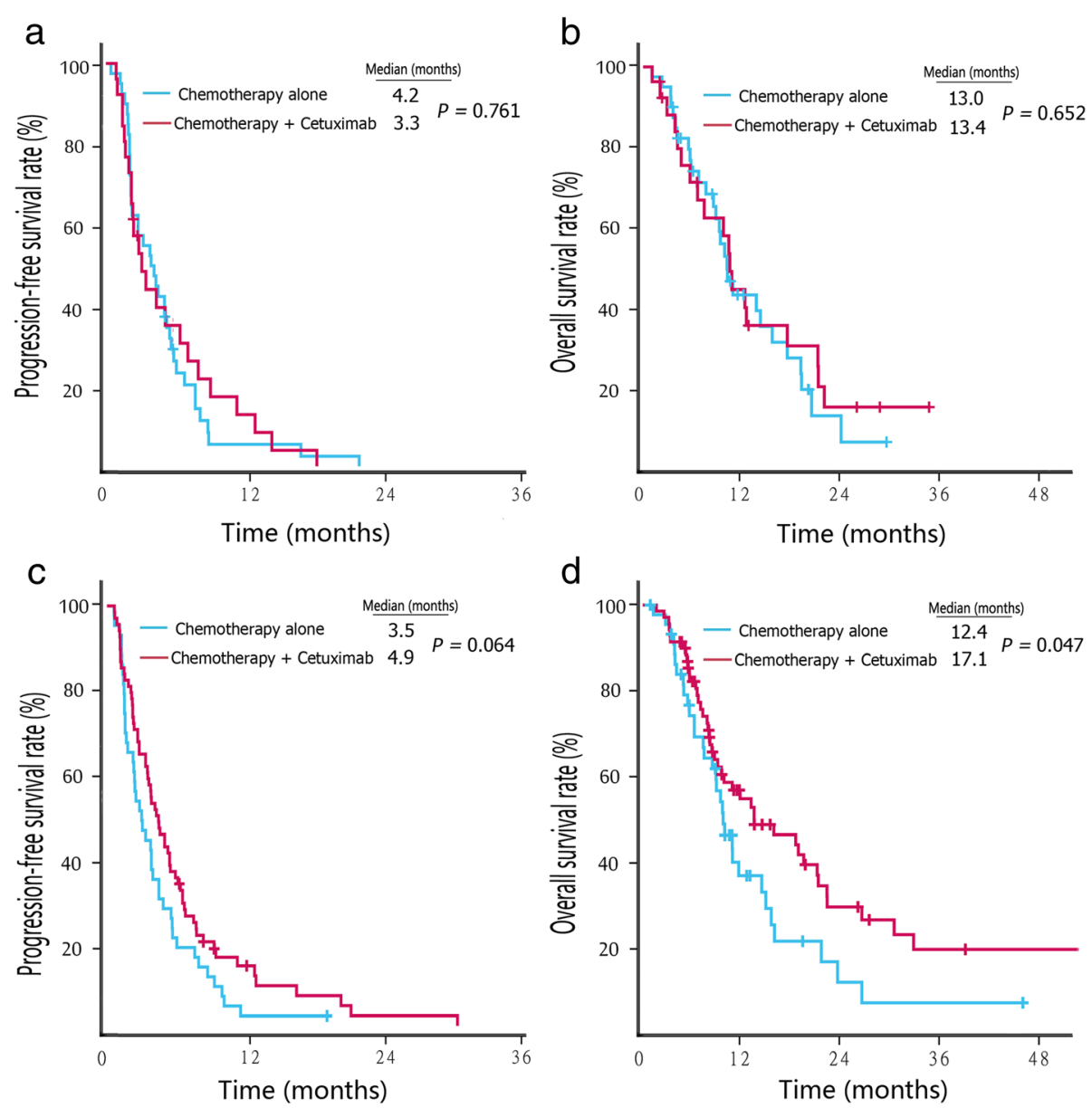

Fig. 2 Kaplan-Meier survival estimates of metastatic colorectal cancer patients with second-line therapy. a, PFS curves of RSCC patients. b, OS curves of RSCC patients. c, PFS curves of LSCRC patients. $\mathbf{d}$, OS curves of LSCRC patients 


\section{PFS and OS}

In RSCC patients who received first-line chemotherapy, the median PFS (5.6 vs. 5.7 months, $P=0.904$ ) (Fig. 1a) and OS (25.1 vs. 19.8 months, $P=0.553$ ) (Fig. 1b) were comparable between those who received cetuximab with chemotherapy and chemotherapy alone. On the other hand, first-line treatment with cetuximab plus chemotherapy resulted in a significantly longer median PFS (9.1 vs. 6.2 months, $P=0.002)$ (Fig. 1 c) and OS (28.9 vs. 20.1 months, $P=0.036$ ) (Fig. 1d) compared with chemotherapy alone in patients with LSCRC.

For second-line therapy, the addition of cetuximab treatment did not prolong the median PFS (3.3 vs. 4.2 months, $P=0.761$ ) (Fig. 2a) or OS (13.4 vs. 13.0 months, $P=0.652$ ) (Fig. 2b) in patients with RSCC. However, in patients with LSCRC, the combination of cetuximab with chemotherapy had a tendency to prolong the median PFS (4.9 vs. 3.5 months, $P=0.064$ ) (Fig. 2 c), and it significantly prolonged the OS (17.1 vs. 12.4 months, $P=$ 0.047) (Fig. 2d).
We also compared the PFS and OS of patients undergoing chemotherapy alone between RSCC and LSCRC groups. No difference in survival was observed between the RSCC and LSCRC patients who received chemotherapy alone as a first-line (PFS, 5.7 vs. 6.2 months, $P=0.160$; OS, 19.8 vs. 20.1 months, $P=0.593$; Fig. $3 \mathrm{a}$ and $\mathrm{b}$ ) or secondline therapy (PFS, 4.2 vs. 3.5 months, $P=0.874$; OS, 13.0 vs. 12.4 months, $P=0.672$; Fig. $3 \mathrm{c}$ and d). Similarly, no difference in survival was observed between the RSCC and LSCRC patients who received cetuximab combined with chemotherapy as a first-line (PFS, 5.6 vs. 9.1 months, $P=$ 0.244; OS, 25.1 vs. 28.9 months, $P=0.512$; Fig. $4 \mathrm{a}$ and b) or second-line therapy (PFS, 3.3 vs. 4.9 months, $P=0.723$; OS, 13.4 vs. 17.1 months, $P=0.120$; Fig. $4 \mathrm{c}$ and d).

\section{Discussion}

The current study showed a significant difference in the ORR and OS between patients with metastatic LSCRC and RSCC who underwent first- or second-line cetuximab treatment. Patients with metastatic LSCRC exhibited a
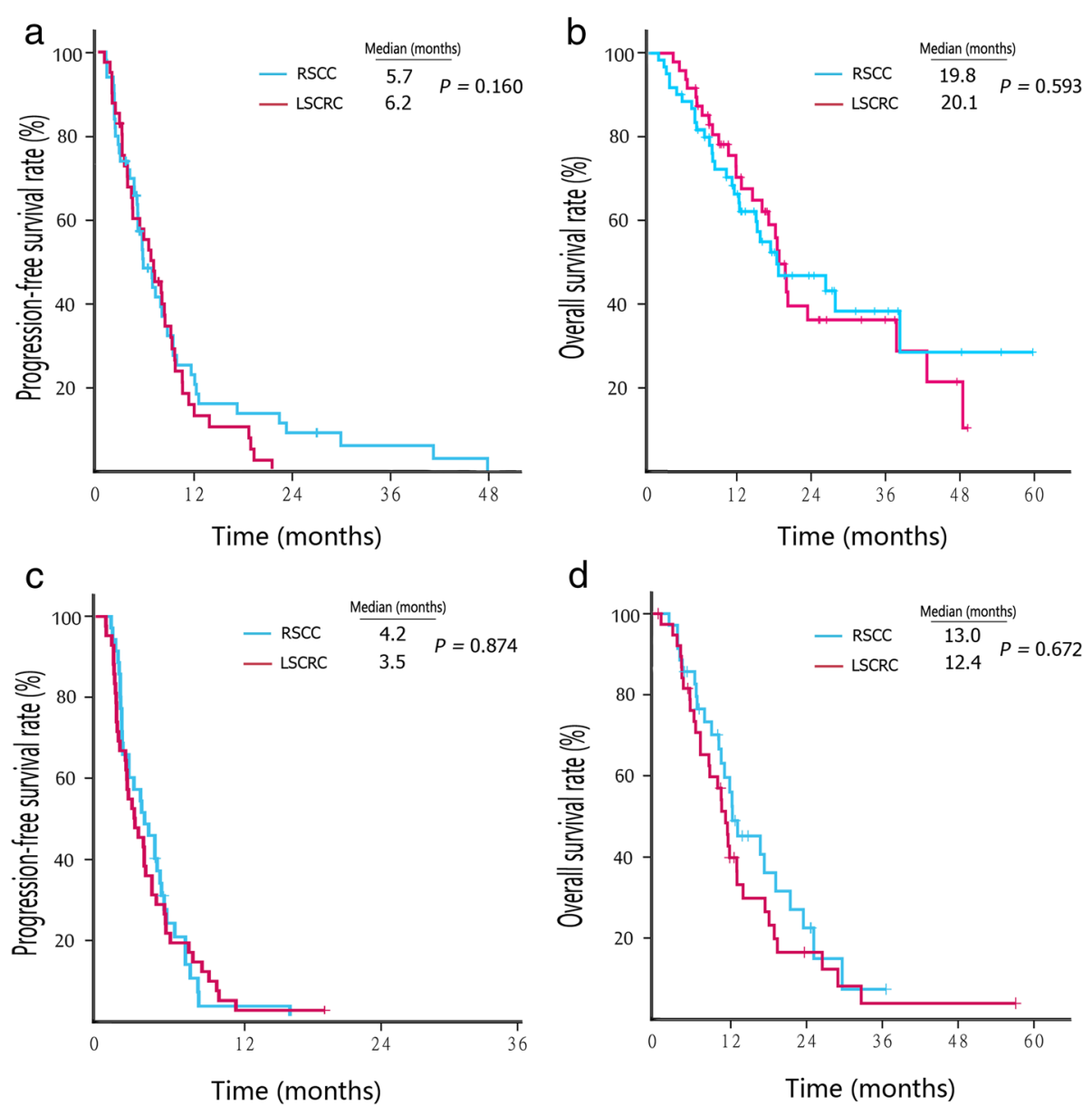

Fig. 3 Kaplan-Meier survival estimates of metastatic colorectal cancer patients treated with chemotherapy alone. a, PFS curves of patients who received first-line therapy. $\mathbf{b}$, OS curves of patients who received first-line therapy. $\mathbf{c}$, PFS curves of patients who received second-line therapy. d, OS curves of patients who received second-line therapy 

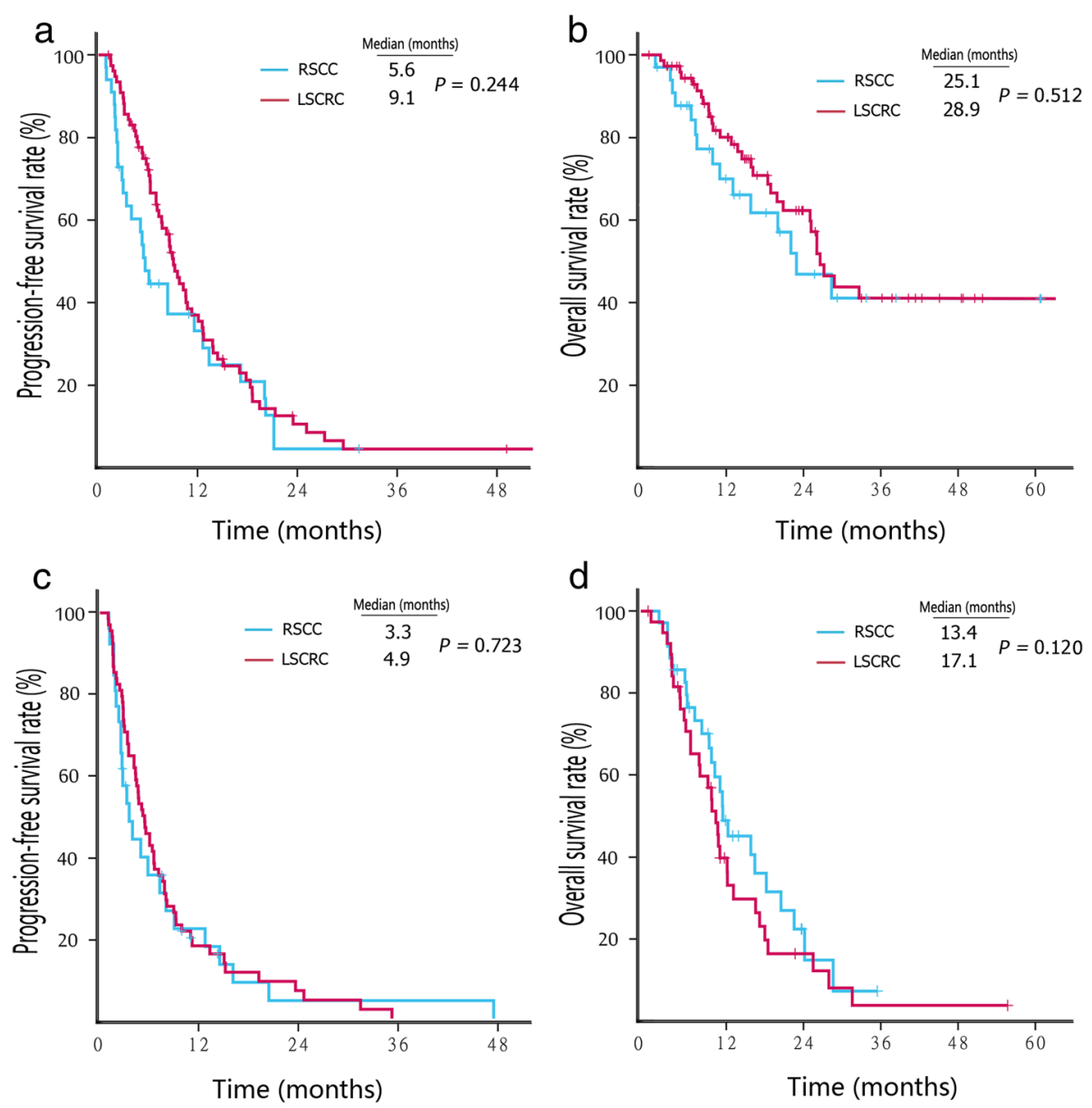

Fig. 4 Kaplan-Meier survival estimates of metastatic colorectal cancer patients treated with cetuximab plus chemotherapy. a, PFS curves of patients who received first-line therapy. $\mathbf{b}$, OS curves of patients who received first-line therapy. $\mathbf{c}$, PFS curves of patients who received second-line therapy. $\mathbf{d}$, OS curves of patients who received second-line therapy

higher response rate to cetuximab plus chemotherapy than to chemotherapy alone, and cetuximab significantly prolonged the PFS and OS in these patients. However, this improvement in the OS or PFS was not observed in patients with RSCC who were treated with cetuximab.

The difference in the response to chemotherapy between LSCRC and RSCC groups was previously reported. However, the current study did not observe a different response to chemotherapy between patients with LSCRC and RSCC. The combination of cetuximab with chemotherapy had a tendency to improve the response rate in LSCRC patients compared with RSCC patients. One study reported that men with right-sided Duke's $C$ colon cancer benefited from adjuvant chemotherapy, but men with left-sided tumors did not [24]. The study also found a significantly higher frequency of MSI in right-sided than in left-sided tumors (20\% vs. $1 \%)$. In the AIO KRK-0104 trial which investigated first-line therapy for mCRC with cetuximab, capecitabine, and irinotecan versus cetuximab, capecitabine, and oxaliplatin, LSCRC was associated with a significantly longer OS and PFS compared with RSCC [25]. A recent study of pre-treated, chemotherapy-refractory mCRC patients showed that cetuximab was associated with a longer PFS compared with best support care in patients with LSCRC (5.4 vs. 1.8 months, $P<0.001$ ), but not in those with RSCC (1.9 vs. 1.9 months, $P=0.26)$ [26].

Although the current study observed an interesting phenomenon, it needs to be confirmed in a randomized prospective study. The underlying mechanisms for the differences in the response rates between LSCRC and RSCC remain unclear. Because the benefit of cetuximab is limited to mCRC patients with wild-type KRAS tumors, the KRAS status in both LSCRC and RSCC needs to be taken into consideration [27]. Several studies found a higher rate of KRAS mutations in RSCC than that in LSCRC $[14,15]$. Other studies found similar KRAS mutation rates in both RSCC and LSCRC $[8,16]$. In addition to KRAS, the BRAF V600E mutation negatively impacts the treatment outcomes for cetuximab in mCRC patients [23]. The BRAF mutation is observed more 
frequently in RSCC than in LSCRC, which might partly explain the higher response rate to cetuximab in patients with LSCRC $[16,28]$. However, the NRAS mutation is mainly found in LSCRC [29]. Alterations of other molecules, including EGFR polysomy, EGFR amplification, and phosphatase and tensin homolog (PTEN) null expression, have been found to be associated with the outcome of cetuximab treatment [30].

\section{Conclusions}

In conclusion, the current study suggests that the primary tumor location may affect the response of patients with mCRC to cetuximab. The addition of cetuximab to first-line and second-line chemotherapy may only benefit patients with metastatic LSCRC. These results should be further confirmed in a prospective study.

\section{Competing interests}

The authors declare that they have no competing interests.

\section{Authors' contributions}

FW conceived of the study and participated in its design and manuscript writing. LB and TSL carried out data collection and statistical analysis. YYY, $\mathrm{MMH}$, and $\mathrm{KYL}$ followed up all the patients. HYL, DSZ, YJ, FHW, ZQW, DSW, $M Z Q$, and $C R$ participated in data collection. YHL and RHX supervised and participated in data analysis and interpretation, and manuscript writing. All authors read and approved the final manuscript.

\section{Acknowledgments}

This work was supported by grants from National Natural Science Foundation of China (No. 81302194 and 81372570), the Specialized Research Fund for the Doctoral Program of Higher Education (No. 20130171120047), and the Fundamental Research Funds for the Central Universities (No.14ykpy40). Feng Wang is a recipient of the Young Talent Fund of Sun Yat-sen University Cancer Center.

\section{Author details}

${ }^{1}$ Department of Medical Oncology, Sun Yat-sen University Cancer Center: State Key Laboratory of Oncology in South China; Collaborative Innovation Center for Cancer Medicine, 651 Dongfeng Road East, Guangzhou, Guangdong 510060, P. R. China. 'Department of Medical Oncology, Zhongshan Hospital, Fudan University, Shanghai 200032, P. R. China

Received: 12 October 2014 Accepted: 8 April 2015

Published online: 10 June 2015

\section{References}

1. Benedix F, Kube R, Meyer F, Schmidt U, Gastinger I, Lippert H. Comparison of 17,641 patients with right- and left-sided colon cancer: differences in epidemiology, perioperative course, histology, and survival. Dis Colon Rectum. 2010:53:57-64.

2. lacopetta B. Are there two sides to colorectal cancer? Int J Cancer. 2002;101:403-8.

3. Fleming M, Ravula S, Tatishchev SF, Wang HL. Colorectal carcinoma: pathologic aspects. J Gastrointest Oncol. 2012:3:153-73.

4. O'Connell JB, Maggard MA, Ko CY. Colon cancer survival rates with the new American Joint Committee on Cancer sixth edition staging. J Natl Cancer Inst. 2004;96:1420-5.

5. Nawa T, Kato J, Kawamoto H, Okada H, Yamamoto H, Kohno H, et al. Differences between right- and left-sided colon cancer in patient characteristics, cancer morphology and histology. J Gastroenterol Hepatol. 2008:23:418-23.

6. Meguid RA, Slidell MB, Wolfgang CL, Chang DC, Ahuja N. Is there a difference in survival between right- versus left-sided colon cancers? Ann Surg Oncol. 2008;15:2388-94.
7. Network CGA. Comprehensive molecular characterization of human colon and rectal cancer. Nature. 2012;487:330-7.

8. Sugai T, Habano W, Jiao YF, Tsukahara M, Takeda Y, Otsuka K, et al. Analysis of molecular alterations in left- and right-sided colorectal carcinomas reveals distinct pathways of carcinogenesis: proposal for new molecular profile of colorectal carcinomas. J Mol Diagn. 2006;8:193-201.

9. Russo A, Bazan V, lacopetta B, Kerr D, Soussi T, Gebbia N. The TP53 colorectal cancer international collaborative study on the prognostic and predictive significance of p53 mutation: influence of tumor site, type of mutation, and adjuvant treatment. J Clin Oncol. 2005;23:7518-28.

10. Nasir A, Kaiser HE, Boulware D, Hakam A, Zhao H, Yeatman T, et al. Cyclooxygenase-2 expression in right- and left-sided colon cancer: a rationale for optimization of cyclooxygenase-2 inhibitor therapy. Clin Colorectal Cancer. 2004;3:243-7.

11. Watatani M, Yoshida T, Kuroda K, leda S, Yasutomi M. Allelic loss of chromosome 17p, mutation of the p53 gene, and microsatellite instability in right- and left-sided colorectal cancer. Cancer. 1996;77:1688-93.

12. Markowitz SD, Bertagnolli MM. Molecular origins of cancer: molecular basis of colorectal cancer. N Engl J Med. 2009;361:2449-60.

13. Hawkins NJ, Tomlinson I, Meagher A, Ward RL. Microsatellite-stable diploid carcinoma: a biologically distinct and aggressive subset of sporadic colorectal cancer. Br J Cancer. 2001;84:232-6.

14. Samowitz WS, Curtin K, Schaffer D, Robertson M, Leppert M, Slattery ML. Relationship of Ki-ras mutations in colon cancers to tumor location, stage, and survival: a population-based study. Cancer Epidemiol Biomarkers Prev. 2000;9:1193-7

15. Bleeker WA, Hayes VM, Karrenbeld A, Hofstra RM, Hermans J, Buys CC, et al. Impact of KRAS and TP53 mutations on survival in patients with left- and right-sided Dukes' C colon cancer. Am J Gastroenterol. 2000;95:2953-7.

16. Hutchins G, Southward K, Handley K, Magill L, Beaumont C, Stahlschmidt J, et al. Value of mismatch repair, KRAS, and BRAF mutations in predicting recurrence and benefits from chemotherapy in colorectal cancer. J Clin Oncol. 2011;29:1261-70.

17. Smith CG, Fisher D, Claes B, Maughan TS, Idziaszczyk S, Peuteman G, et al. Somatic profiling of the epidermal growth factor receptor pathway in tumors from patients with advanced colorectal cancer treated with chemotherapy +/- cetuximab. Clin Cancer Res. 2013;19:4104-13.

18. Mendelsohn J, Baselga J. The EGF receptor family as targets for cancer therapy. Oncogene. 2000;19:6550-65.

19. Yang $X$, Zhang $X$, Mortenson ED, Radkevich-Brown O, Wang Y, Fu YX. Cetuximab-mediated tumor regression depends on innate and adaptive immune responses. Mol Ther. 2013;21:91-100.

20. Broadbridge VT, Karapetis CS, Price TJ. Cetuximab in metastatic colorectal cancer. Expert Rev Anticancer Ther. 2012;12:555-65.

21. Karapetis CS, Khambata-Ford S, Jonker DJ, O'Callaghan CJ, Tu D, Tebbutt NC et al. K-ras mutations and benefit from cetuximab in advanced colorectal cancer. N Engl J Med. 2008;359:1757-65.

22. Lievre A, Bachet JB, Le Corre D, Boige V, Landi B, Emile JF, et al. KRAS mutation status is predictive of response to cetuximab therapy in colorectal cancer. Cancer Res. 2006;66:3992-5.

23. Di Nicolantonio F, Martini M, Molinari F, Sartore-Bianchi A, Arena S, Saletti $P$, et al. Wild-type BRAF is required for response to panitumumab or cetuximab in metastatic colorectal cancer. J Clin Oncol. 2008;26:5705-12.

24. Elsaleh H, Joseph D, Grieu F, Zeps N, Spry N, lacopetta B. Association of tumour site and sex with survival benefit from adjuvant chemotherapy in colorectal cancer. Lancet. 2000;355:1745-50.

25. von Einem JC, Heinemann V, von Weikersthal LF, Vehling-Kaiser U, Stauch M, Hass HG, et al. Left-sided primary tumors are associated with favorable prognosis in patients with KRAS codon 12/13 wild-type metastatic colorectal cancer treated with cetuximab plus chemotherapy: an analysis of the AIO KRK-0104 trial. J Cancer Res Clin Oncol. 2014;140:1607-14

26. Brule SY, Jonker DJ, Karapetis CS, O'Callaghan CJ, Moore MJ, Wong R, et al. Location of colon cancer (right-sided [RC] versus left-sided [LC]) as a predictor of benefit from cetuximab (CET): NCIC CTG CO.17. J Clin Oncol. 2013;31:3528. abstr.

27. Van Cutsem E, Kohne CH, Hitre E, Zaluski J, Chang Chien CR, Makhson A, et al. Cetuximab and chemotherapy as initial treatment for metastatic colorectal cancer. N Engl J Med. 2009;360:1408-17.

28. Tie J, Gibbs P, Lipton L, Christie M, Jorissen RN, Burgess AW, et al. Optimizing targeted therapeutic development: analysis of a colorectal 
cancer patient population with the BRAF (V600E) mutation. Int J Cancer. 2011;128:2075-84.

29. Irahara N, Baba Y, Nosho K, Shima K, Yan L, Dias-Santagata D, et al. NRAS mutations are rare in colorectal cancer. Diagn Mol Pathol. 2010;19:157-63.

30. Laurent-Puig P, Cayre A, Manceau G, Buc E, Bachet JB, Lecomte T, et al. Analysis of PTEN, BRAF, and EGFR status in determining benefit from cetuximab therapy in wild-type KRAS metastatic colon cancer. J Clin Oncol. 2009:27:5924-30

Submit your next manuscript to BioMed Central and take full advantage of:

- Convenient online submission

- Thorough peer review

- No space constraints or color figure charges

- Immediate publication on acceptance

- Inclusion in PubMed, CAS, Scopus and Google Scholar

- Research which is freely available for redistribution 